\section{Elimination of Dialysis in the Preparation of Hydrophobic Sols}

THe preparation of hydrophobic colloidal solutions by double decomposition, as, for example,

$$
\mathrm{AgNO}_{3}+\mathrm{KI} \rightarrow \mathrm{AgI}+\mathrm{KNO}_{3}
$$

is greatly complicated by the elaborate process of dialysis necessary to remove the unwanted electrolyte, in this case potassium nitrate. The sols purified by electrodialysis usually contain a considerablo quantity of polyvalent ions, drawn from the water used in the dialysis into the colloidal solution. These ionic contaminants are strongly adsorbed to the sol particles and it is often difficult to eliminate them.

The following procedure, tested in our laboratory with good results, avoids the formation of a foreign electrolyte and therefore avoids subsequent dialysis.

A cation exchange resin ('Dowex' 50) was loaded with 200 m.equiv. silver ion by treating the hydrogenexchange resin with a solution of $200 \mathrm{~m}$.mol. of silver nitrate.

$$
R \mathrm{H}+\mathrm{AgNO}_{3} \rightarrow R \mathrm{Ag}+\mathrm{HNO}_{3}
$$

In order to eliminate the electrolyte (nitric acid) the resin was washed several times with distilled water.

Then 1 litre of solution containing $200 \mathrm{~m} . \mathrm{mol}$. of potassium iodide was added to the silver resin; at once a colloidal solution of silver iodide was formed.

$$
R \mathrm{Ag}+\mathrm{KI} \rightarrow R \mathrm{~K}+\mathrm{AgI}
$$

The reaction being complete, a highly concentrated sol (about 200 m.mol. silver iodide/l.) was obtained in one operation.

Conductivity measurements showed that it contains very little electrolyte $\left(\sigma=3 \cdot 1 \times 10^{-5} \mathrm{ohm}^{-1} \mathrm{~cm} .^{-1}\right)$, which compares favourably with the degree of purity of electrodialysed sols ; the risk of contamination of the sol by polyvalent ions was excluded. The stability of the sols thus prepared was also excellent.

This sol, which we called a potassium sol (the counter-ions of the sol particles are formed by potassium ions), can be converted in a hydrogen sol or a barium sol by sending it over a cation-exchanger saturated with hydrogen or barium ions.

Physical Chemistry Laboratory,

P. NAGELS

University of Ghent. Nov. 20.

\section{Separation, Detection and Estimation of C2I 17 :20-Dihydroxy-20-methyl-steroids}

A NUMBER of C21 steroids characterized by the 17 : 20-dihydroxy-20-methyl side-chain at C17 have been isolated from the urine of normal people ${ }^{1,2}$ and from the urine of patients with adrenocortical hyperplasia ${ }^{8}$ or adrenocortical tumours ${ }^{4}$ (see Table 1). The separation and identification of members of this group of steroids is necessary for the study of their normal and abnormal metabolic formation and exeretion; the following is a method which is capable of separating, detecting and estimating quantitatively all such urinary steroids so far isolated.

The known steroids of this series are detectable on dried paper chromatograms using the 70 per cent phosphoric acid test described for pregnane-3 $\alpha: 17 \alpha$ : $20 \alpha$-triol-11-one $\theta^{5}$ and pregnane-3 $\alpha: 17 \alpha: 20 \alpha$-triol ${ }^{6}$. The results of the colour test on the $17: 20$.dihydroxy20-methyl-steroids are shown in Table 1. An approximate estimate of the amounts of such steroids on a paper chromatogram may be obtained from the intensity of the colour or fluorescence and the size of the spots. The relative sensitivity of the colour test for the different steroids is indicated in the table; amounts of up to about ten times the minimum can be judged from the intensity and size of spot.

Some of the compounds in Table 1 have been separated on paper chromatographic systems of the Zaffaroni type ${ }^{6}$ (compounds I and V) or the Bush typ $\theta^{2}$ (compounds I, II, III). Paper chromatographic studies of mixtures of all the steroids I-VI have shown that complete separation of the compounds is possible in a descending chromatogram, run with the solvent system benzene/formamide for $12 \mathrm{hr}$. on a 35-cm. long Whatman No. 1 paper at $25^{\circ} \mathrm{C}$. The rates of movement of the steroids relative to pregnane$3 \alpha: 17 \alpha: 20 \alpha$-triol are shown in Table 1. Compounds II and IV move closely together, but under the above conditions separate completely ; the largest amounts of each steroid used in these tests corresponded to about $12 \mu \mathrm{gm} . / \mathrm{cm}$. width of paper. Mixed with neutral chloroform extracts, prepared from urine treated with $\beta$-glucuronidase preparations for hydrolysis of steroid conjugates, compounds I-VI continued to give satisfactory chromatographs and moved at the same relative rates as were found with the pure steroids.

A general method for the estimation of $17: 20$ dihydroxy-20-methyl-steroids and its application to urinary extracts has been described ? ; this method is based on the formation of acetaldehyde from the C17 side-chain of these steroids ('acetaldehydogenic steroids') when oxidized with periodic acid. As steroids with other C17 side-chains are not estimated and do not interfere in this estimation, it is particularly suitable for the assay of the steroids listed in Table 1, after their separation by paper chromatography. It has been found that this estimation of 'acetaldehydogenic steroids' may be carried out without modification directly on dried portions of paper chromatograms (benzene/formamide system) ; elution of the steroids from the paper prior to oxida. tion is not necessary. Amounts of steroid down to about $2 \mu \mathrm{gm}$. can be estimated quantitatively on the paper.

Table 1. SEParation AND Detectron of $17: 20$-Dikydroxy-20-Metey S Steroids

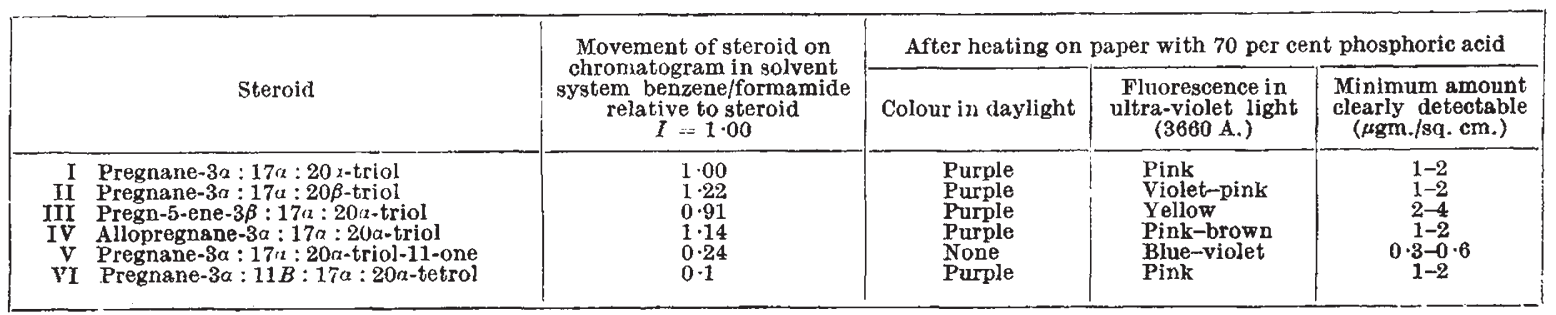

